\title{
Ein Flohmarktfund
}

Jürgen Elstrodt

Bei einem Bummel über den Flohmarkt von Münster fiel mein Blick auf einen jungen Verkäufer, der mir bekannt vorkam. Er sprach mich auch gleich an: Guten Tag, Herr Professor. Interessieren Sie sich immer noch für Geschichte der Mathematik? Verblüfft konnte ich nur ein gedehntes Jaaa ... erwidern, da fuhr mein Gesprächspartner fort: Sie haben uns doch in der Vorlesung Ihr Manuskript von Riemann gezeigt. Wollen Sie eine Postkarte von Weierstraß? Da erkannte ich in dem jungen Mann einen Hörer meiner letzten Vorlesung über Funktionentheorie. In der Lehrveranstaltung hatte ich mein Autograf von Riemann (s. [4]) gezeigt, in dem die Cauchy-Riemannschen Differentialgleichungen von Riemanns Hand niedergeschrieben sind. Der Anblick hatte bei vielen Studierenden einen nachhaltigen Eindruck hinterlassen.

Im weiteren Verlauf des Gesprächs stellte sich heraus: Der Anbieter hatte einen kleinen Stapel alter Postkarten aus dem letzten Viertel des 19. Jahrhunderts erworben zum Weiterverkauf an Philatelisten. Beim Durchblättern des Stapels bemerkte er einige mathematische Formeln auf einer Karte, entzifferte die Unterschrift und erkannte den Namen „Weierstraß“. Natürlich war ich am Erwerb der Karte interessiert, aber der forsch geforderte Betrag von 60 Mark erschien mir utopisch: Einem armen alten Professor wollen Sie 60 Mark aus der Tasche ziehen für 'ne olle Postkarte? - Für Sie 30!, war die Antwort, und der Handel war abgeschlossen.

\section{Die Karte}

Die Karte aus hellbraunem Karton hat das alte Format von $14 \times 9 \mathrm{~cm}$, das auf dem Weltpostkongress in Paris 1878 festgelegt wurde. Das Porto von 5 Pfennigen ist aufgedruckt; ausgegeben wurde die Karte von der Deutschen Reichspost. Der Poststempel trägt das Datum „27. I. [18]79“; zugestellt wurde die Karte am gleichen Tage.

Postkarten wurden ab dem I. Juli 1870 vom Generalpostmeister Heinrich Stephan im damaligen Norddeutschen Bund eingeführt als praktisches Medium für kurze Mitteilungen, und diese Neuerung wurde rasch ein durchschlagender Erfolg. Postkarten aus dieser Zeit entwickelten sich später zu einem beliebten Sammelobjekt.

Auf der Vorderseite der Karte befindet sich die Anschrift:

Herrn Professor $D^{r}$ Roethig

in Berlin, $\mathrm{S}$.

Oranienstr. |3|

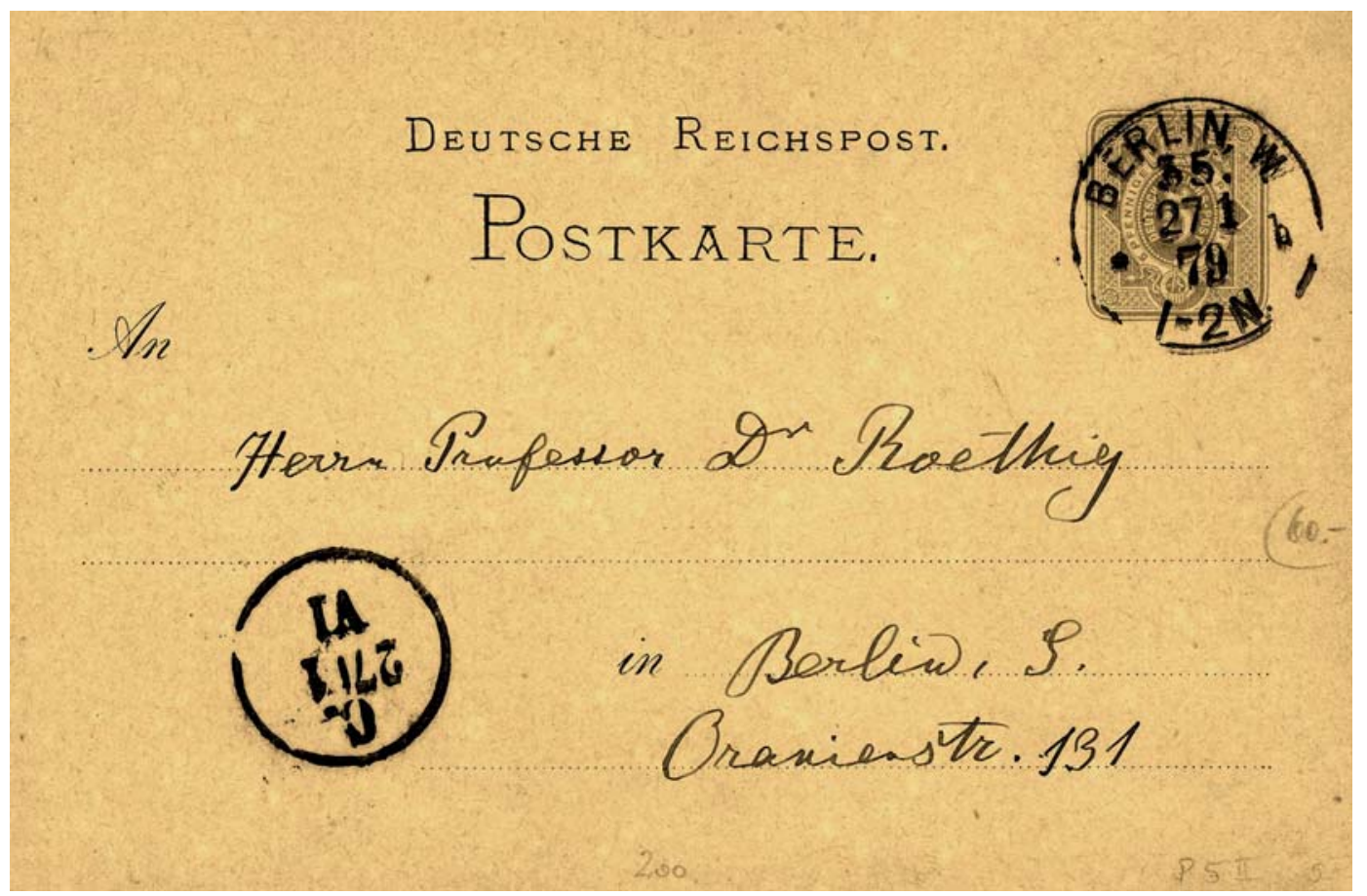




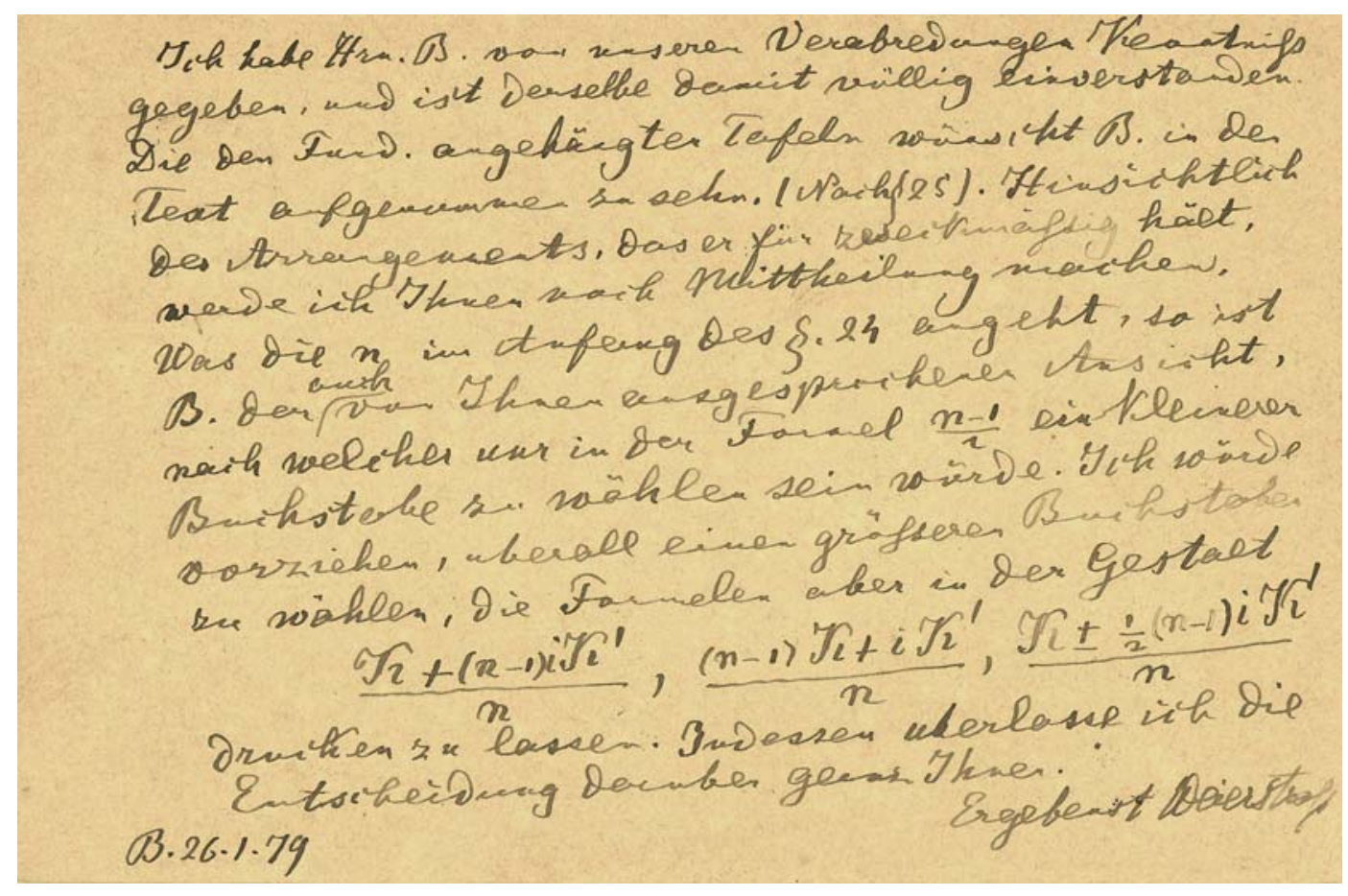

\section{Der Text auf der Rückseite lautet:}

Ich habe $\mathrm{H}[\mathrm{er}] \mathrm{rn} \mathrm{B}$ [orchardt] von unseren Verabredungen Kenntniß gegeben, und ist derselbe damit völlig einverstanden. Die den Fund[amenta nova theoriae functionum ellipticarum] angehängten Tafeln wünscht $B$ [orchardt] in den Text aufgenommen zu sehn. (Nach § 25). Hinsichtlich des Arrangements, das er für zweckmäßig hält, werde ich Ihnen noch Mitteilung machen. Was die $n$ im Anfang des $\$ .24$ angeht, so ist $\mathrm{B}$ [orchardt] der auch von Ihnen ausgesprochenen Ansicht, nach welcher nur in der Formel $\frac{n-1}{2}$ ein kleinerer Buchstabe zu wählen sein würde. Ich würde vorziehen, überall einen größeren Buchstaben zu wählen, die Formeln aber in der Gestalt $\frac{K+(n-1) i K^{\prime}}{n}, \frac{(n-1) K+i K^{\prime}}{n}, \frac{K \pm \frac{1}{2}(n-1) i K^{\prime}}{n}$ drucken zu lassen. Indessen überlasse ich die Entscheidung darüber gerne Ihnen.

B[erlin] 26.I.[18]79

Ergebenst Weierstraß

\section{Historischer Kontext}

Die Karte wurde am 26. I. 1879 geschrieben und bezieht sich auf die Herausgabe der Gesammelten Werke von Carl Gustav Jacob Jacobi (I804-185I). Zur fraglichen Zeit war Weierstraß' enger Freund Carl Wilhelm Borchardt (1817-1880) im Auftrage der Königlich PreuBischen Akademie der Wissenschaften mit der Edition der Werke Jacobis betraut (s. [7], S. I30 f.).
Borchardt war ein Doktorand und Freund Jacobis, hatte sich 1848 in Berlin habilitiert und durch eigene Arbeiten und die Herausgabe des Journals für die reine und angewandte Mathematik ein hohes Ansehen in der Fachwelt erworben. Daher war er für seine Aufgabe ,wie kein anderer befähigt und berufen", wie Weierstraß in seiner Vorrede zum ersten Band von Jacobis Werken schreibt ([6, $\mathrm{S}$. VI]). Wegen seines labilen Gesundheitszustands konnte Borchardt aber in den letzten Lebensjahren seine Arbeit als Herausgeber nicht mehr in vollem Umfang wahrnehmen; er starb am 27.6. 1880 in Rüdersdorf bei Berlin. Das Datum auf der Karte belegt, dass Weierstraß bereits zu diesem frühen Zeitpunkt die Edition der Werke Jacobis gemeinsam mit Borchardt geleitet hat. Noch zwei Monate vor seinem Tode konnte Borchardt allerdings seine Ausarbeitung von Jacobis Vorlesung ,Theorie der elliptischen Functionen aus den Eigenschaften der Thetareihen abgeleitet" für den Druck vorbereiten (s. [6, S. 497-538 und S. 545]). In einem Brief vom 28. 10. 1880 an seine Schülerin Sofja Kowalewskaja (1850-189I) teilt Weierstraß dann mit:

Nach Borchardts Erkrankung mußte ich die Herausgabe von Jacobi fortführen, da sich eine dazu geeignete Person dazu nicht fand - und ich habe selbst früher nicht geglaubt, daß ein solches Unternehmen so viel zeitraubende Arbeit erfordert. ([2, S. 236 f.])

Auf dem Titelblatt des 188I erschienenen ersten Bandes von Jacobis Gesammelten Werken ist Borchardt als Herausgeber benannt, und in der Vorrede würdigt Weierstraß mit warmen Worten die Verdienste seines ,verewigten 


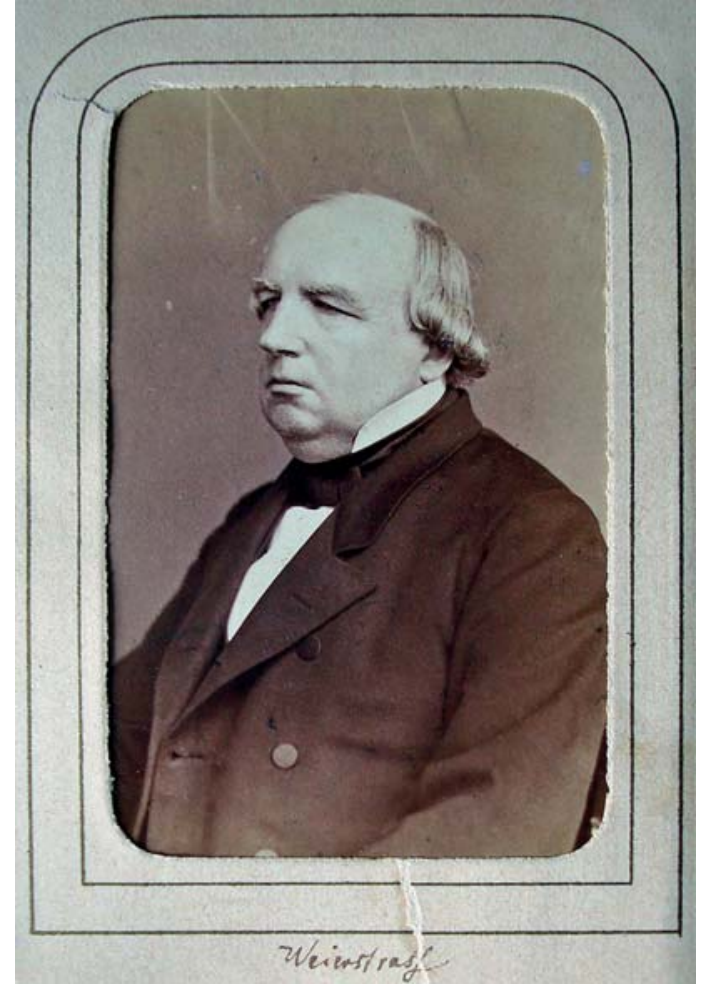

Freundes" bei der Edition. Überdies dankt er zahlreichen weiteren Fachkollegen für ihre Hilfe. Unter den Namen befindet sich auch Oscar Roethig [oft „Röthig“ geschrieben], der beim Lesen der Korrekturen geholfen hatte.

\section{Der Adressat}

Johann Wilhelm Oscar Röthig (1834-1903) studierte nach dem Abitur von 1854 bis 1857 Mathematik und Naturwissenschaften an der Berliner Friedrich-WilhelmsUniversität. Er erwarb 1857 den Doktorgrad der Philosophischen Fakultät mit der auf Anregung von Ernst Eduard Kummer (1810-1893) angefertigten Dissertation De quibisdam generibus integralium ellipticorum ([I, S. 35I]). Da Weierstraß ab 1856 neben seiner Professur am Königlichen Gewerbe-Institut zusätzlich eine besoldete außerordentliche Professur an der Friedrich-WilhelmsUniversität innehatte, ist es gut denkbar, dass er Röthig bereits damals kennengelernt hat. Im Jahre 1858 erwarb Röthig bei der wissenschaftlichen Prüfungskommission in Berlin die facultas docendi für die Fächer Mathematik und Physik. Im gleichen Jahr wurde er Hülfslehrer an der Städtischen Gewerbschule zu Berlin. Diese Lehranstalt wurde in der Folge mehrfach umbenannt. Sie hieß ab 1866 die Friedrichs-Werdersche Gewerbeschule und ab 1872 (offiziell ab 1882) Friedrichs-Werdersche OberRealschule und setzte sich zum Ziel, ihren Absolventen nach zunächst acht, später neun Schuljahren ,eine möglichst vollkommene Vorbildung für alle Studien auf Tech-

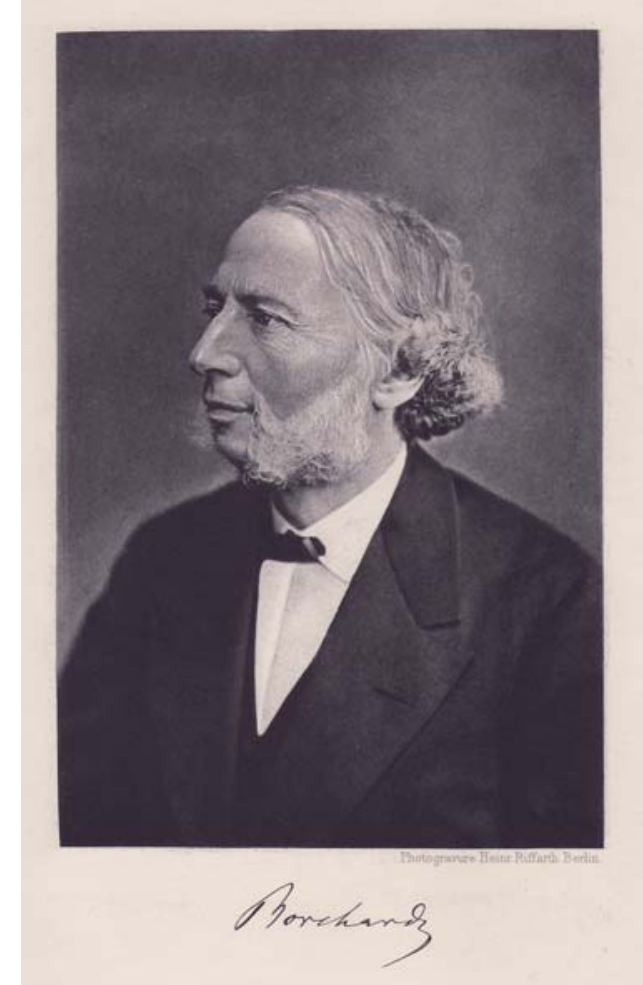

nischen Hochschulen zu geben“, wie Röthig als stellvertretender Direktor im Jahresbericht 1890/9I der Lehranstalt schrieb. Nach Beförderungen zum ordentlichen Lehrer (1860) und Oberlehrer (1863) wurde Röthig im Jahre 1876 in Anerkennung seiner Verdienste der Titel Professor verliehen. Röthig trat 1900 in den Ruhestand und starb 1903.

\section{Typografische Spuren}

Weierstraß' Karte an Röthig bezieht sich auf die typografische Gestaltung des Texts der Fundamenta [5] im ersten Band von Jacobis Werken [6]. Es ist eine bemerkenswerte Fügung, dass ausgerechnet Weierstraß mit der Edition dieses Werks befasst war, das ihm zur Zeit seines Selbststudiums der Mathematik in Bonn (1836-1838) zunächst unüberwindliche Schwierigkeiten bereitet hatte. Erst nachdem ihm ein Kommilitone die Nachschrift einer Vorlesung des münsterschen Mathematikers Christoph Gudermann (1798-|85I) über elliptische Funktionen ausgeliehen hatte, konnte er die Fundamenta verstehen und gleich eigene Untersuchungen vornehmen ([3, S. 2I]).

Zunächst geht Weierstraß in seinem Schreiben auf die „den Fund. angehängten Tafeln“ ein. Dabei handelt es sich um folgendes drucktechnisches Problem: Die Seiten der Originalausgabe [5] der Fundamenta haben das Format $26 \times 20,5 \mathrm{~cm}$, aber zwischen den Seiten 56 und 57 ist nach $\S 24$ eine mehrfach gefaltete große Tafel von $44 \times 43,5$ 
cm mit zahlreichen Formeln zur Transformation der elliptischen Funktionen eingefügt. In der Überschrift der Tafel weist der Zusatz ,(\$.25.)“ darauf hin, dass die Tafeln zu $§ 25$ gehören. In den Originalexemplaren der Fundamenta wurden diese Tafeln vermutlich einzeln von Hand eingeklebt oder den ungebundenen Exemplaren für den Buchbinder lose beigefügt. Um Probleme beim Druck von [6] zu vermeiden, verabredeten Borchardt und Weierstraß, die Tafeln in den Text aufzunehmen, und zwar „Nach § 25“, wie Weierstraß schreibt, unter dem Vorbehalt, dass er über das „Arrangement ...noch Mitteilung machen" werde. Ein Blick in [6] lehrt, dass die Tafeln nach $\S 27$, S. 116-119 abgedruckt wurden.

Ferner unterbreitet Weierstraß Vorschläge zur typografischen Gestaltung der Formeln in [5], S. 49, am Anfang von $§ 24$. Beispielhaft zitiert er auf der Karte nur den jeweils letzten Term der fraglichen drei Formelzeilen. Diese Terme schreibt er in besonders schöner Handschrift mit sorgfältigen Schnörkeln. Der Grund dafür könnte folgender sein: In [5] wird sowohl für den Text als auch für die Formeln durchweg die Schrift Antiqua verwendet. Nur recht selten erscheinen z. B. Eigennamen, neu eingeführte Begriffe oder ausformulierte Theoreme in Kursivschrift. Beim Druck von [6] hingegen wurden die im mathematischen Schriftsatz üblichen Regeln befolgt, denen zufolge z. B. Buchstaben in Formeln kursiv zu setzen sind, allgemein übliche Funktionssymbole wie $\sin , \cos , \log$, sin am etc. jedoch in Antiqua. Weierstraß' Handschrift scheint sich an dieser Regel zu orientieren. Die letzte der auf der Karte genannten Formeln erscheint im Druck in [6, S. 100], jedoch (abweichend von Weierstraß' Vorschlag) in der Form

$$
\frac{K \pm \frac{n-1}{2} i K^{\prime}}{n}
$$

wobei für den Bruch $\frac{n-1}{2}$ eine etwas kleinere Schrift benutzt wird, wie von Borchardt und Röthig vorgesehen.

\section{Resümee}

Vergleicht man den mit zahlreichen Fehlern behafteten Text der Fundamenta [5] mit dem Text der Gesammelten Werke [6], so kann man nur die große Sorgfalt bewundern, welche die Herausgeber haben walten lassen. Manche Editionen gesammelter Werke aus neuerer Zeit, in denen die Originaltexte fotomechanisch ohne Korrektur der Errata reproduziert wurden, schneiden dagegen überaus schlecht ab.

Nach Erscheinen des ersten Bandes [6] war Weierstraß noch zehn Jahre lang mit der Herausgabe der übrigen sechs Bände befasst. Am 25. 10. I891 konnte er dann an Jacobis Witwe Marie, geb. Schwinck (1807-1901) schreiben (s. [9, S. 522]):
Es gereicht mir zur besondern Freude, Ihnen mitteilen zu können, daß nunmehr auch der letzte Band der von der Academie veranstalteten Gesammtausgabe der Werke Ihres verewigten Gatten fertig geworden ist. In 7 stattlichen Bänden ist jetzt, systematisch nach Gegenständen geordnet und möglichst correct gedruckt, alles vereinigt und den weitesten Kreisen zugänglich gemacht, was der große Mathematiker auf dem Gebiete seiner Wissenschaft als Forscher geleistet hat... Sie dürfen es mit mir als das schönste Monument betrachten, welches seinem unsterblichen Urheber hätte gesetzt werden können.

\section{Literatur}

[I] Biermann, Kurt-R.: Die Mathematik und ihre Dozenten an der Berliner Universität 1810-1933. Berlin: Akademie-Verlag 1988.

[2] Bölling, Reinhard (Hrsg.): Briefwechsel zwischen Karl Weierstraß und Sofja Kowalewskaja. Berlin: Akadmie-Verlag 1993.

[3] Elstrodt, Jürgen: Die prägenden Jahre im Leben von Karl Weierstraß. In [8], S. II-5I.

[4] Elstrodt, Jürgen u. Ullrich, Peter: A real sheet of complex Riemannian function theory: A recently discovered sketch in Riemann's own hand. Hist. Math. 26, 268-288 (1999).

[5] Jacobi, Carl Gustav Jacob: Fundamenta nova theoriae functionum ellipticarum. Regiomonti (= Königsberg): Fratres Borntraeger 1829.

[6] Jacobi, Carl Gustav Jacob: Gesammelte Werke. Erster Band. Berlin: G. Reimer I88I.

[7] Knobloch, Eberhard: Weierstraß und die Preußische Akademie der Wissenschaften. In [8], S. I23-141.

[8] König, Wolfgang u. Sprekels, Jürgen (Hrsg.): Karl Weierstraß (I815-1897). Aspekte seines Lebens und Werkes. Wiesbaden: Springer Spektrum 2016.

[9] Koenigsberger, Leo: Carl Gustav Jacob Jacobi. Leipzig: B. G. Teubner 1904.

Prof. Dr. Jürgen Elstrodt, Mathematisches Institut, Universität Münster, Einsteinstraße 62, 48I49 Münster elstrod@uni-muenster.de

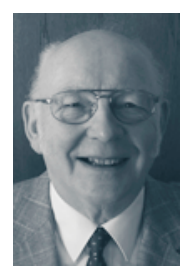

Jürgen Elstrodt (geb. 1940), Studium der Mathematik und Physik 1959-1966 U Münster und LMU München; Promotion 1970, Habilitation 1974, wiss. Rat und Prof. 1974 LMU München; seit 1976 Prof. U Münster, ab 2005 Ruhestand. Arbeitsgebiete: reelle und komplexe Analysis, automorphe Funktionen, Geschichte der Mathematik. Bücher (z. T. als Koautor): Maß- und Integrationstheorie, Harmonische Analysis auf dem dreidimensionalen hyperbolischen Raum, Geschichte der Mathematik an der U Münster; Mitherausgeber des Buchs „Die elliptischen Funktionen und ihre Anwendungen. Dritter Teil" "von Robert Fricke. 